\title{
Probing natural SUSY from stop pair production at the LHC
}

\author{
Junjie $\mathrm{Cao}^{1,2}$, Chengcheng $\mathrm{Han}^{3}$, Lei $\mathrm{Wu}^{3}$, Jin Min Yang ${ }^{3}$, Yang Zhang ${ }^{1,3}$ \\ 1 Physics Department, Henan Normal University, Xinxiang 45300\%, China \\ 2 Center for High Energy Physics, Peking University, Beijing 100871, China \\ 3 Institute of Theoretical Physics, Academia Sinica, Beijing 100190, China
}

\begin{abstract}
We consider the natural supersymmetry scenario in the framework of the $R$-parity conserving minimal supersymmetric standard model (called natural MSSM) and examine the observability of stop pair production at the LHC. We first scan the parameters of this scenario under various experimental constraints, including the SM-like Higgs boson mass, the indirect limits from precision electroweak data and B-decays. Then in the allowed parameter space we study the stop pair production at the LHC followed by the stop decay into a top quark plus a lightest neutralino or into a bottom quark plus a chargino. From detailed Monte Carlo simulations of the signals and backgrounds, we find the two decay modes are complementary to each other in probing the stop pair production, and the LHC with $\sqrt{s}=14 \mathrm{TeV}$ and $100 \mathrm{fb}^{-1}$ luminosity is capable of discovering the stop predicted in natural MSSM up to $450 \mathrm{GeV}$. If no excess events were observed at the LHC, the $95 \%$ C.L. exclusion limits of the stop masses can reach around $537 \mathrm{GeV}$.
\end{abstract}

PACS numbers: 14.80.Da,14.80.Ly,12.60.Jv 


\section{INTRODUCTION}

Although the standard model (SM) has been successful in describing the existing experimental data, it is suffering from the hierarchy problem and new physics based on certain symmetry is widely expected to appear at $\mathrm{TeV}$ scale to stabilize the electroweak hierarchy against radiative corrections. This belief was further strengthened by the recent discovery of the Higgs boson at the Large Hadron Collider (LHC) with its mass determined around $125 \mathrm{GeV}$ [1]. This mass value agrees well with the prediction of low energy supersymmetry (SUSY), which is so far the most promising new physics candidate.

In SUSY, all known bosons and fermions have their supersymmetric partners, and the scalar top quarks (called stop $\tilde{t}_{i}$ with $i=1,2$ ), as the top quark partners, can modify the property of the SM Higgs boson by exactly canceling out the dangerous quadratic divergence of the top quark loop. Obviously, the experimental determination of the stop properties is crucial to unravel the nature of supersymmetry in protecting the Higgs mass at the weak scale and thus solving the hierarchy problem. In fact, such activities have been carried out extensively at the hadronic colliders such as the LHC and the Tevatron [2 4], but in contrast with the strong mass bounds (about $1 \mathrm{TeV}$ ) on the gluino and the first generation squarks [5], a relatively light stop (say about $300 \mathrm{GeV}$ ) can not be excluded. Nevertheless, it should be mentioned that the recently measured Higgs boson mass around $125 \mathrm{GeV}$ may give some indications for the stop sector $[6-8]$. In the popular MSSM with moderate $\tan \beta$ and large $m_{A}$, the Higgs mass is given by [6]

$$
m_{h}^{2} \simeq M_{Z}^{2} \cos ^{2} 2 \beta+\frac{3 m_{t}^{4}}{4 \pi^{2} v^{2}} \ln \frac{M_{S}^{2}}{m_{t}^{2}}+\frac{3 m_{t}^{4}}{4 \pi^{2} v^{2}} \frac{X_{t}^{2}}{M_{S}^{2}}\left(1-\frac{X_{t}^{2}}{12 M_{S}^{2}}\right),
$$

where $v=174 \mathrm{GeV}, X_{t} \equiv A_{t}-\mu \cot \beta$ and $M_{S}$ is the average stop mass scale defined by $M_{S}=\sqrt{m_{\tilde{t}_{1}} m_{\tilde{t}_{2}}}$. This expression indicates that, for the heavier stop $\tilde{t}_{2}$ around $1 \mathrm{TeV}$ as discussed above, the lighter stop $\tilde{t}_{1}$ must be heavier than about $200 \mathrm{GeV}$ and $\left|X_{t}\right|$ must be larger than $1.5 \mathrm{TeV}$ in order to push the Higgs mass up to $125 \mathrm{GeV}$ [8]. About these constraints, one should keep in mind that they are independent of the decay modes of $\tilde{t}_{1}$, but on the other hand, they may be greatly weakened if there exists additional contribution to the Higgs mass [8, 9].

On the theoretical side, there are good reasons to consider at least one stop significantly lighter than other squarks with a mass around several hundred GeV. Firstly, in some popular grand unification models, supersymmetry breaking is usually assumed to transmit to 
the visible sector at a certain high energy scale, and then Yukawa contributions to the renormalization group evolution tend to reduce stop masses more than other squark masses. Secondly, the chiral mixing for certain flavor squarks is proportional to the mass of the corresponding quark, and is therefore more sizable for stops. Such a mixing will further reduce the mass of the lighter stop. Thirdly, in the MSSM the minimization conditions of its Higgs potential imply [10]

$$
\frac{M_{Z}^{2}}{2}=\frac{\left(m_{H_{d}}^{2}+\Sigma_{d}\right)-\left(m_{H_{u}}^{2}+\Sigma_{u}\right) \tan ^{2} \beta}{\tan ^{2} \beta-1}-\mu^{2},
$$

where $m_{H_{d}}^{2}$ and $m_{H_{u}}^{2}$ represent the weak scale soft SUSY breaking masses of the Higgs fields, $\mu$ is the higgsino mass parameter, $\tan \beta \equiv v_{u} / v_{d}$. $\Sigma_{u}$ and $\Sigma_{d}$ arise from the radiative corrections to the Higgs potential and the dominant contribution to the $\Sigma_{u}$ is given by

$$
\Sigma_{u} \sim \frac{3 Y_{t}^{2}}{16 \pi^{2}} \times m_{\tilde{t}_{i}}^{2}\left(\log \frac{m_{\tilde{t}_{i}}^{2}}{Q^{2}}-1\right) .
$$

These two equations indicate that, if the individual terms on the right hand side of Eq. (2) are comparable in magnitude so that the observed value of $M_{Z}$ is obtained without resorting to large cancelations, the natural values of $\mu$ and $m_{\tilde{t}_{i}}$ should be around $100 \mathrm{GeV}$ and several hundred $\mathrm{GeV}$ respectively. Numerically, the requirement of $\Sigma_{u}<M_{Z}^{2} / 2$ (or $\Sigma_{u}<v^{2}$ ) leads to $m_{\tilde{t}_{1,2}}$ upper bounded by about $500 \mathrm{GeV}$ (or $1.5 \mathrm{TeV}$ ) [11]. Moreover, we note that a light stop is also phenomenologically needed by the electroweak baryogenesis [12] and may be welcomed by the dark matter physics [13]. In the MSSM, although the gluino contribute to $m_{\tilde{t}_{i}}^{2}$ at the one-loop level and to $m_{H_{u}}^{2}$ at two-loop level, the corrections are proportional to $m_{\tilde{g}}^{2}$ and can be greatly enhanced by the large gluino mass[14]. In order to the keep the naturalness, we expect $m_{\tilde{g}}$ to be lighter than about $3 \mathrm{TeV}$ for $m_{\tilde{t}}<1.5 \mathrm{TeV}$. However, since the current results of searching for the supersymmetry indicate that a gluino with mass about $1 \mathrm{TeV}$ can safely avoid the LHC constraints, we require $1 \mathrm{TeV}<m_{\tilde{g}}<3 \mathrm{TeV}$ in our calculation.

Motivated by the theoretical preference and the results of the LHC search for SUSY, recently the natural MSSM scenario attracted broad attention [14 18], which focuses on the following parameter space of the MSSM [14, 17]:

- $|\mu| \lesssim 100-200 \mathrm{GeV}$ and $m_{\tilde{t}_{1,2}} \leq 1-1.5 \mathrm{TeV}$ as preferred by Eq.(22) and Eq.(3) ; 
- $1 \mathrm{TeV} \lesssim m_{\tilde{g}} \lesssim 3-4 \mathrm{TeV}$ to escape the LHC constraint and at same time to avoid spoiling color symmetry; while the electroweak-ino masses may still be at sub-TeV scale;

- $m_{A} \sim\left|m_{H_{d}}^{2}\right|^{\frac{1}{2}} \lesssim|\mu| \tan \beta$ as suggested by the relation $m_{A}^{2} \simeq 2 \mu^{2}+m_{H_{u}}^{2}+m_{H_{d}}^{2}+\Sigma_{u}+\Sigma_{d}$ and Eq.(2);

- $m_{\tilde{q}_{1,2}}, m_{\tilde{l}_{1,2}} \sim 10-50 \mathrm{TeV}$ to provide a decoupling solution to the SUSY flavor and CP problems.

Since the stop are relatively light and sensitive to probing this scenario, there have been recently many theoretical studies on the collider signatures of the light directly produced stop in the $R$-parity conserving and violating MSSM[19]. For example, by using the top tagging technique, the sensitivity of stop searches were studied in the hadronic, semi-leptonic and di-leptonic channels[20 23]. In order to suppress the di-leptonic top backgrounds, the authors in Ref.[24] explore some new kinematic observables developed from $M_{T_{2}}$ to improve the sensitivity of the stop searches. For the small mass splitting between stop and top, it is pointed that the rapidity difference and spin correlation of the daughter products from stops decay can be helpful to discover the signal[25]. When the stop mass is close to the lightest supersymmetric particle mass, the monojet signature from $\tilde{t}_{1} \tilde{t}_{1}^{*}+j$ production is expected to be useful in detecting the stop[26]. If the stop mass is degenerate with the sum of the masses of its decay products, the searches based on missing transverse energy $\left(E_{T}^{\text {miss }}\right.$ or $\left.\mathbb{E}_{T}\right)$ have significant reach for stop masses above $175 \mathrm{GeV}[27]$. When the $R$-parity is violated, the decay modes of the stop will be very different from the ones in $R$-parity conserving MSSM, such as stop decaying to dilepton and trilepton final states[28]. We also noted that the constrains on the light stop in the natural SUSY have been discussed by using the results from sparticles searches at the LHC, and indicated that they were mild and can be safely avoided currently [14, 17, 18, 29].

In this work, we investigate the potential of the LHC in probing the lighter stop $\tilde{t}_{1}$ predicted by the natural MSSM with $R$-parity, which is based on some considerations: (i) Most of the studies of the stop searches have been carried out under some assumptions at the LHC in a model independent way or in simplified models. It will be meaningful to explore what might happen in a realistic model like MSSM under the current available experimental constraints; (ii) Due to the $R$-parity conservation, there will be sizeable missing 
energy appearing in the sparticles productions and decays, which can be easily identified in the LHC data; (iii) One interesting phenomenological feature of the natural MSSM with $R$-parity is that both the lightest neutralino and the lighter chargino are Higgsino-like, and consequently $\tilde{t}_{1}$ always decays dominantly into $t \tilde{\chi}_{1}^{0}$ and $b \tilde{\chi}_{1}^{+}$with $\tilde{\chi}_{1}^{+} \rightarrow \tilde{\chi}_{1}^{0} W^{*}$, which can greatly simplify the analysis of the $\tilde{t}_{1}$ detection at the LHC.

For this purpose, we first scan the parameter space of the natural MSSM by considering various constraints in Sec. II. Then in Sec. III we discuss the observability of $\tilde{t}_{1}$ through the direct stop pair production in the allowed parameter space by performing the Monte Carlo simulations for the channel $p p \rightarrow \tilde{t}_{1} \tilde{t}_{1}^{*} \rightarrow t \tilde{\chi}_{1}^{0} \bar{t}_{\bar{\chi}}^{0}$ and the channel $p p \rightarrow \tilde{t}_{1} \tilde{t}_{1}^{*} \rightarrow b \tilde{\chi}_{1}^{+} \bar{b} \tilde{\chi}_{1}^{-}$. We will present their corresponding sensitivities for $8 \mathrm{TeV}$ LHC and for $14 \mathrm{TeV}$ LHC respectively. Finally in Sec. IV, we summarize the conclusions obtained in this work.

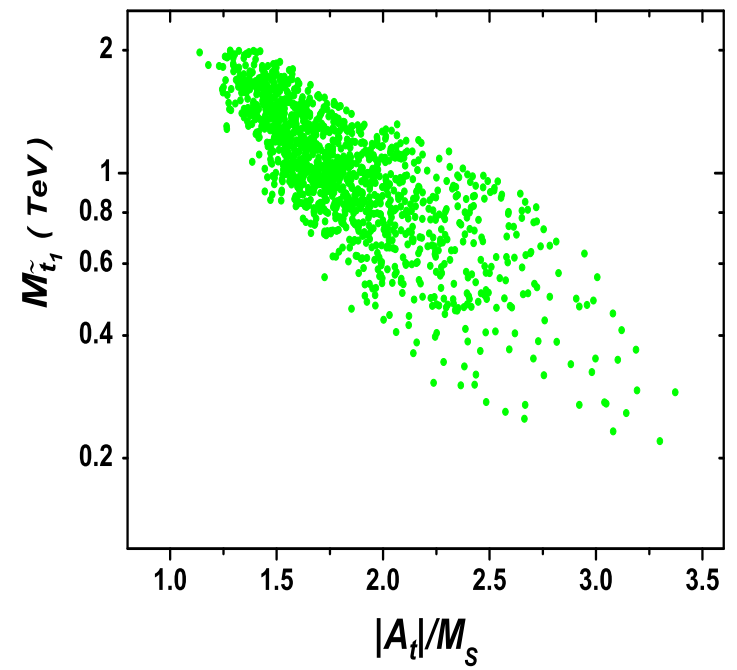

FIG. 1: Scatter plot of surviving samples in natural MSSM, projected on the plane of $m_{\tilde{t}_{1}}$ versus $\left|A_{t}\right| / M_{S}$. All the samples here predict the SM-like Higgs boson in the mass range $125 \pm 2 \mathrm{GeV}$.

\section{SCAN OVER THE PARAMETER SPACE}

Motivated by the natural MSSM, we scan the parameter space of the MSSM as follows:

$$
\begin{aligned}
& 1 \leq \tan \beta \leq 60, \quad 100 \mathrm{GeV} \leq \mu \leq 200 \mathrm{GeV}, \quad 100 \mathrm{GeV} \leq \mathrm{M}_{2} \leq 1 \mathrm{TeV} \\
& \left|A_{t}\right| \leq 3 \mathrm{TeV}, \quad 100 \mathrm{GeV} \leq\left(\mathrm{M}_{\tilde{\mathrm{Q}}_{3}}, \mathrm{M}_{\tilde{\mathrm{U}}_{3}}\right) \leq 2 \mathrm{TeV}, \quad 90 \mathrm{GeV} \leq \mathrm{M}_{\mathrm{A}} \leq 1 \mathrm{TeV}
\end{aligned}
$$




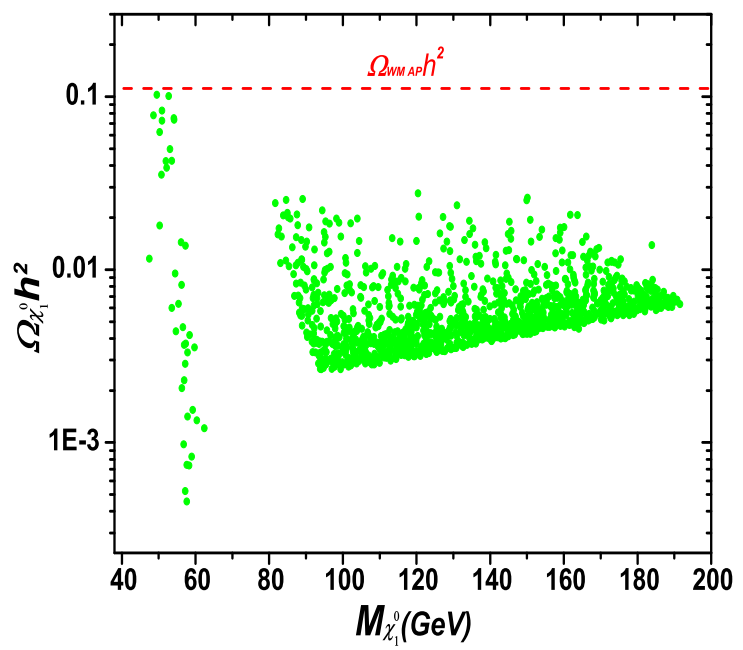

FIG. 2: Same as Fig.1, but showing the relic density of the neutralino dark matter.

For other unimportant parameters, we fix all the soft breaking parameters in the slepton sector and the first two generation sector at $10 \mathrm{TeV}$, and we assume $A_{t}=A_{b}, M_{\tilde{U}_{3}}=M_{\tilde{D}_{3}}$ and $M_{1}: M_{2}=1: 2$ (inspired by the grand unification relation). In our scan, we consider following constraints:

- We require the SM-like Higgs mass within the range $125 \pm 2 \mathrm{GeV}$. We use the code FeynHiggs2.8.6 [30] to calculate the mass and the code HiggsBounds-3.8.0 [31] to consider the experimental constraints on the Higgs sector of the natural MSSM.

- Since the natural MSSM has important implications in B-physics [32], we use the code susy_flavor v2.0 [33] to consider the constraints from the processes $B \rightarrow X_{s} \gamma$ and $B_{s(d)} \rightarrow \mu^{+} \mu^{-}$.

- We consider indirect constraints from the precision electroweak observables such as $\rho_{l}$, $\sin ^{2} \theta_{\text {eff }}^{l}, m_{W}$ and $R_{b}$. We use our own code for such calculation [34].

- We require the thermal relic density of the lightest neutralino (as the dark matter candidate) is below the WMAP value [35]. We use the code MicrOmega v2.4 [36] to calculate the density.

After analyzing the surviving samples, we find they have two characters. One is that the Higgs mass of $125 \pm 2 \mathrm{GeV}$ requires $m_{\tilde{t}_{1}} \geq 220 \mathrm{GeV}$ and there is a rather strong correlation 


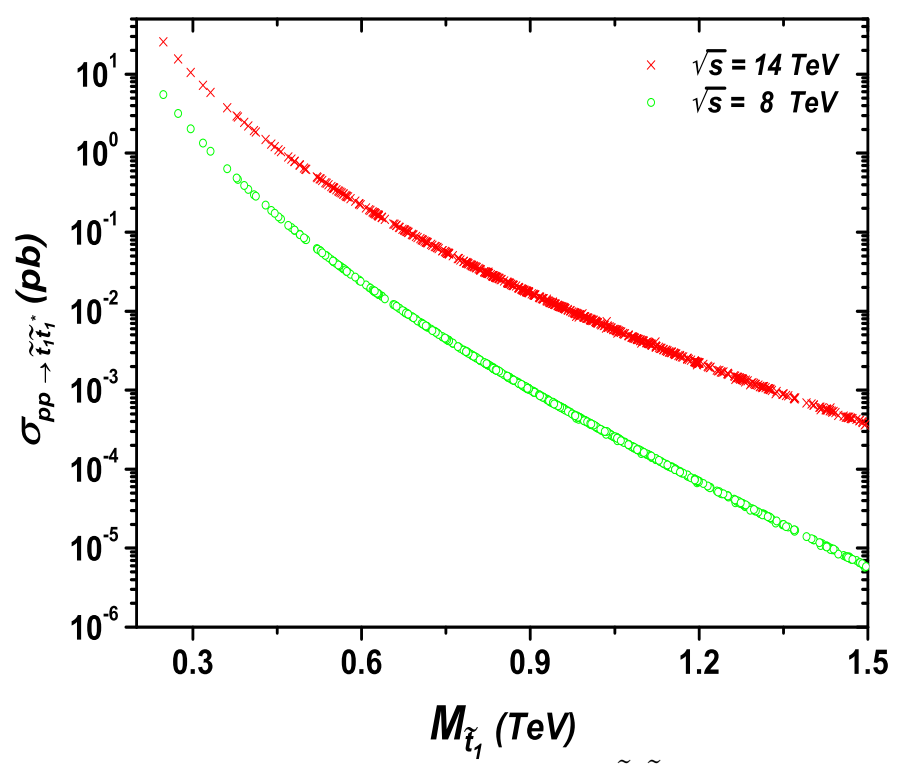

FIG. 3: Same as Fig,1, but showing the cross section of $\tilde{t}_{1} \tilde{t}_{1}^{*}$ production versus the stop mass.

between $m_{\tilde{t}_{1}}$ and the ratio $\left|A_{t}\right| / M_{S}$, as shown in Fig.1. Here we further clarify that, if $M_{\tilde{\Omega}_{3}}$ and $M_{\tilde{U}_{3}}$ are at sub-TeV scale, the minimum of $m_{\tilde{t}_{1}}$ will be enhanced to about $300 \mathrm{GeV}[8]$. The other feature is that for most cases, the values of $\mu$ are significantly smaller than $M_{1}$ so that the lightest neutralino is higgsino-like. Fig.2 indicates that the surviving samples lie within two isolated regions. We checked that the lightest neutralino is bino-like in the left region and higgsino-like in the right region. Here the bino(higgsino)-like means it is still a mixed state but the dominant component is bino(higgsino). For the light neutralino dark matter(bino-like), the main annihilation channel is through exchanging $\mathrm{Z}$ boson. The annihilation cross section is roughly proportional to $1 /\left(4 m_{\chi}^{2}-m_{Z}^{2}\right)^{2}$. When the neutralino mass is about $50 \mathrm{GeV}-60 \mathrm{GeV}$, the annihilation cross section may be very large, so the relic density will be less than 0.1 . When the neutralino becomes heavier $(60 \mathrm{GeV}-90 \mathrm{GeV}$, neutralino is still bino-like), the annihilation cross section will drop. The relic density becomes large and even exceeds the WMAP value, and these samples are excluded. This is the reason for the gap between $60 \mathrm{GeV}-90 \mathrm{GeV}$. When the neutralino goes on becoming heavy $(>90 \mathrm{GeV})$, the dominant component of the neutralino will be higgsino. The coupling between neutralino and Higgs gets important and annihilation rate goes up, then the relic density drops.

About the natural MSSM, we have two comments. One is that in this scenario the diphoton signal of the SM-like Higgs boson can hardly be enhanced to satisfy the requirement of the LHC data. This is because in the framework of the MSSM, there are only two cases 
which can enhance the di-photon rate, i.e. the small $\alpha_{\text {eff }}$ scenario [37, 38] and the light $\tilde{\tau}$ scenario [6, 39], and in each case a large $\mu$ is needed. In the Ref.[40], the authors pointed that the light stop with large couplings to Higgs boson in the $\mathrm{SM}+$ stop model can improve the SM fitting to the LHC and Tevatron data by enhancing $\Gamma(h \rightarrow \gamma \gamma)$ and suppressing $\Gamma(h \rightarrow g g)$. However, we should note that it does not mean that the di-photon production rate can reach the measurement of the LHC in a concrete MSSM model since the reduction of $\Gamma(h \rightarrow g g)$ is usually much stronger than the enhancement of $\Gamma(h \rightarrow \gamma \gamma)$ for large values of $X_{t}\left(X_{t}=A_{t}-\mu \tan \beta\right)[41]$. The other is that recently the ATLAS collaboration searched for the gluino-mediated stop pair production followed by the decay $\tilde{t}_{1} \rightarrow b \tilde{\chi}_{1}^{+} \rightarrow b \ell \nu \tilde{\chi}_{1}^{0}$, which set a lower bound $m_{\tilde{t}_{1}} \geq 450 \mathrm{GeV}$ [3]. This conclusion is not applicable to the our calculations since we take the gluino mass to be larger than $1 \mathrm{TeV}$ in the allowed parameters space of the natural SUSY.

\section{OBSERVABILITY OF STOP PAIR PRODUCTION AT THE LHC}

In this section we discuss the LHC potential of discovering the stop through the direct stop pair production in the natural MSSM at $\sqrt{s}=8,14 \mathrm{TeV}$. In Fig 3 we show the $p p \rightarrow \tilde{t}_{1} \tilde{t}_{1}^{*}$ production rate at the next leading order for the surviving samples. In getting this figure we used the package Prospino2.1 [42] and the parton distribution function CTEQ6.6m [43] with the renormalization scale $\mu_{R}$ and factorization scale $\mu_{F}$ setting to $m_{\tilde{t}_{1}}$. This figure indicates that the maximal values of the cross section reach $5.5 \mathrm{pb}$ and $25.7 \mathrm{pb}$ for the LHC with $\sqrt{s}=8 \mathrm{TeV}$ and $\sqrt{s}=14 \mathrm{TeV}$ respectively, and with the increase of the stop mass, the production rates drop rapidly.

In Fig, 4 we present various decay branching ratios of $\tilde{t}_{1}$ which are obtained by using the package SDECAY [44]. This figure indicates that for $m_{\tilde{t}_{1}} \leq 320 \mathrm{GeV}$ where the decay channel $\tilde{t}_{1} \rightarrow t \tilde{\chi}_{1}^{0}$ does not open up, $\tilde{t}_{1}$ decays into $b \tilde{\chi}_{1}^{+}$with a ratio of $100 \%$, and as the stop becomes heavier, the branching ratios for $\tilde{t}_{1} \rightarrow b \tilde{\chi}_{1}^{+}$and $\tilde{t}_{1} \rightarrow t \tilde{\chi}_{1}^{0}$ may still be around $50 \%$. In contrast, the branching ratios for $\tilde{t}_{1}$ decays into $t \tilde{\chi}_{3,4}^{0}$ and $b \tilde{\chi}_{2}^{+}$are usually less than $20 \%$.

In the following we perform detailed Monte Carlo simulations to investigate the observability of the direct stop pair production at the LHC. We concentrate on the semi-leptonic analysis with the b-tagging efficiency $40 \%$, where the signal is consisted of four jets(at least 

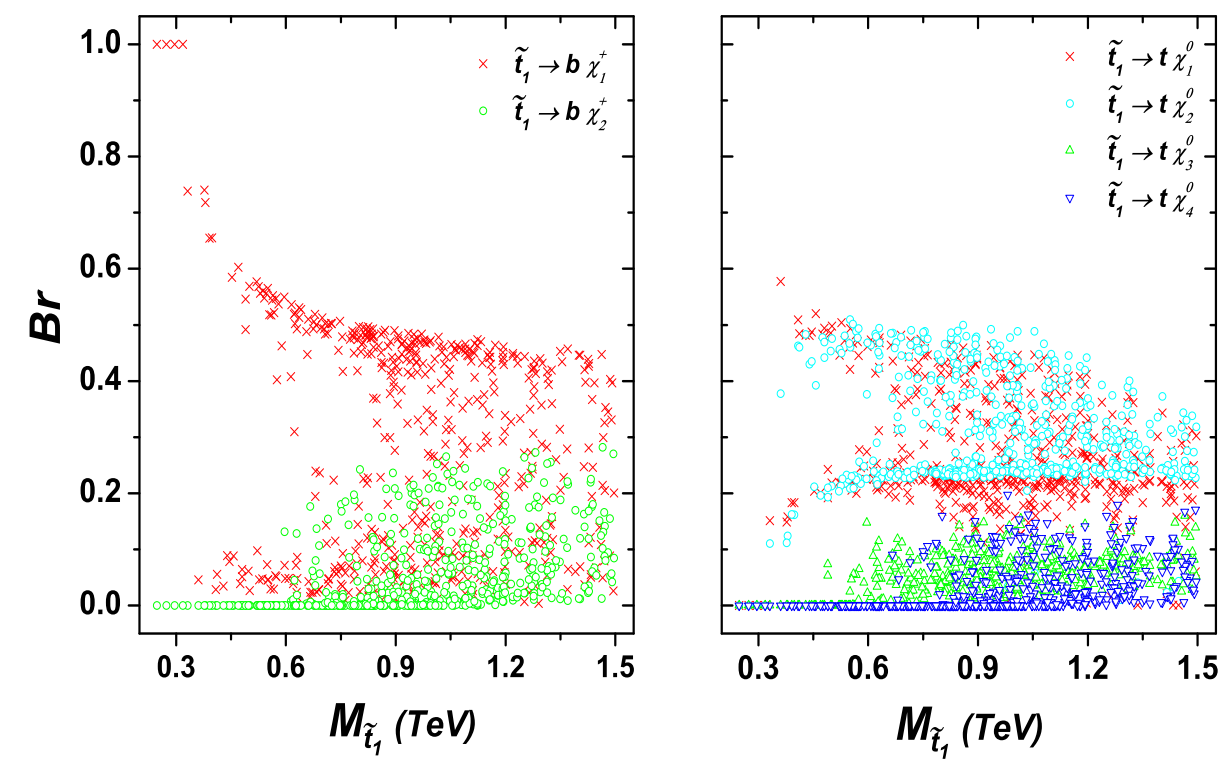

FIG. 4: Same as Fig, but showing the decay branching ratios of the stop.

one b-jet), one lepton ( $e$ or $\mu$ ), and missing transverse energy. We first consider the process

$$
p p \rightarrow \tilde{t}_{1} \tilde{t}_{1}^{*} \rightarrow\left(t \tilde{\chi}_{1}^{0}\right)\left(\bar{t} \tilde{\chi}_{1}^{0}\right) \rightarrow\left(b \ell^{+} \nu \tilde{\chi}_{1}^{0}\right)\left(\bar{b} j j \tilde{\chi}_{1}^{0}\right) \text { or }\left(\operatorname{bjj} \tilde{\chi}_{1}^{0}\right)\left(\bar{b} \ell^{-} \bar{\nu} \tilde{\chi}_{1}^{0}\right)
$$

From the ATLAS search for the signal $t \bar{t}+E_{T}^{m i s s}$ [2], we can see that the dominant SM background after the $E_{T}^{\text {miss }}$ and $M_{T}$ cuts is $t \bar{t}$ di-leptonic channel with one lost lepton and two additional jets from initial state radiation to fake the hadronic $W$. Another backgrounds include $t \bar{t}$ semi-leptonic channel, $t \bar{t}$ di-leptonic channel with one $\tau$ from top decay misidentified as a jet, $W+$ jets and $t \bar{t} Z$. Here we emphasize that the $t \bar{t} Z$ background becomes important for a heavy stop and should be considered in estimating the significance. In our calculation, we normalize the signal and the $t \bar{t}$ background to their NLO values [42, 45], and simulate the signal and backgrounds by MadGraph5 [46] interfaced with PYTHIA [47] and Delphes [48] to carry out the parton shower and fast detector simulation. We use the anti- $k_{t}$ algorithm [49] with the distance parameter $R=0.4$ to cluster jets and the MLM scheme [50] to match our matrix element with parton shower. We checked that the shapes of the matched $W+1,2,3$ partons are very similar, and for simplicity, we take $W+2$ jets samples in our calculations. In our calculations, since we employ the the variable $M_{T 2}^{W}$ defined in Ref.[24], we checked our results with theirs for the same parameters at $\sqrt{s}=7 \mathrm{TeV}$ and found they were consistent with each other.

In Fig.5, we show the distributions of $\mathbb{E}_{T}$, the transverse mass $M_{T}$ defined in [2] and $M_{T 2}^{W}$ for the backgrounds and our benchmark point $m_{\tilde{t}_{1}}=429 \mathrm{GeV}$ and $m_{\tilde{\chi}_{1}^{0}}=110 \mathrm{GeV}$ 

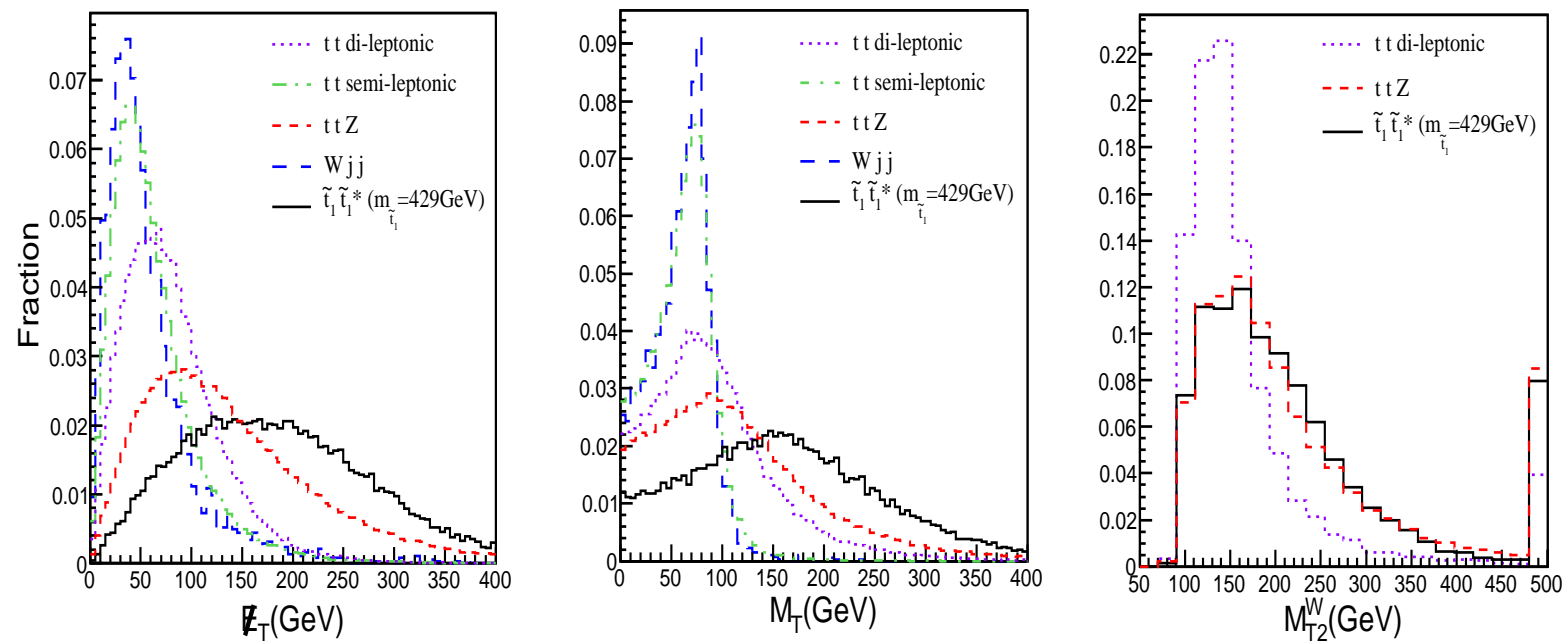

FIG. 5: The distributions of $\ell+4$-jets $+\mathbb{E}_{T}$ with respect to $\mathbb{E}_{T}, M_{T}$ and $M_{T 2}^{W}$ for the signal $p p \rightarrow$ $\tilde{t}_{1} \tilde{t}_{1}^{*} \rightarrow t \tilde{\chi}_{1}^{0} \bar{t} \tilde{\chi}_{1}^{0}$ and backgrounds. In the $M_{T 2}^{W}$ distribution, we impose the cuts $\mathbb{E}_{T}>150 \mathrm{GeV}$ and $M_{T}>150 \mathrm{GeV}$ on the events and only display the di-leptonic $t \bar{t}, t \bar{t} Z$ backgrounds and signal, where the events with wrong or no solution for $M_{T 2}^{W}$ are included in the last bin.

TABLE I: The significance of stop pair production $p p \rightarrow \tilde{t}_{1} \tilde{t}_{1}^{*} \rightarrow t \tilde{\chi}_{1}^{0} \bar{t} \tilde{\chi}_{1}^{0}$ for $100 f b^{-1}$ luminosity after imposing various cuts. Here we take $m_{\tilde{t}_{1}}=429 \mathrm{GeV}$ and $\tilde{\chi}_{1}^{0}=110 \mathrm{GeV}$ for illustration.

\begin{tabular}{|c|c|c|c|c|}
\hline $\mathbb{E}_{T^{- \text {cut }}(\mathrm{GeV})}$ & $M_{T^{- \text {cut }}(\mathrm{GeV})}$ & $M_{T 2}^{W}$-cut $(\mathrm{GeV})$ & $S / \sqrt{B}(8 \mathrm{TeV})$ & $S / \sqrt{B}(14 \mathrm{TeV})$ \\
\hline 150 & - & - & 1.26 & 4.05 \\
\hline 150 & 150 & - & 2.75 & 7.91 \\
\hline 150 & 150 & 173 & 3.11 & 8.60 \\
\hline
\end{tabular}

with $\sqrt{s}=8 \mathrm{TeV}$ (similar results are found for $\sqrt{s}=14 \mathrm{TeV}$ ). This figure indicates that most events of $W+\mathrm{jj}$ and semi-leptonic $t \bar{t}$ backgrounds are characterized by $\mathbb{E}_{T} \leq 100 \mathrm{GeV}$ and $M_{T} \leq 100 \mathrm{GeV}$, and most events of the di-leptonic $t \bar{t}$ backgrounds are characterized by $M_{T 2}^{W} \leq 170 \mathrm{GeV}$, while a significant fraction of the signal may have larger $\mathbb{E}_{T}, M_{T}$ and $M_{T 2}^{W}$. Fig. 5 also indicates that the distributions of the $t \bar{t} Z$ background are quite similar to the signal and are difficult to be suppressed. Fortunately, the production rate of $t \bar{t} Z$ is much smaller than the one of $\tilde{t}_{1} \tilde{t}_{1}^{*}$.

In Table I, we present the significance $S / \sqrt{B}$ of our benchmark point for $100 \mathrm{fb}^{-1}$ luminosity with $\sqrt{s}=8 \mathrm{TeV}$ and $14 \mathrm{TeV}$ respectively by sequentially imposing the cuts on $\mathbb{E}_{T}$, $M_{T}$ and $M_{T 2}^{W}$. It can be seen that, for the given reference point, the cut $M_{T}>150 \mathrm{GeV}$ can 
greatly enhance the significance and $M_{T 2}^{W}>173 \mathrm{GeV}$ further improves the significance by about $14 \%$ for $\sqrt{s}=8 \mathrm{TeV}$ and $9 \%$ for $\sqrt{s}=14 \mathrm{TeV}$ to reach 3.11 and 8.60 respectively.

Therefore, for our simulations in the allowed parameters space, we take the following events selection criteria:

- One isolated electron or muon that passes the following requirements;

- Electrons $E_{T}>25 \mathrm{GeV}$ and $|\eta|<2.47$ without $1.37<|\eta|<1.52$;

- Muon: $E_{T}>20 \mathrm{GeV}$ and $|\eta|<2.5$;

- Events are rejected if they contain a second lepton candidate with $P_{T}>15 \mathrm{GeV}$;

- Four or more reconstructed jets with $P_{T}>25 G e V$ and $|\eta|<2.5$.

- $\mathbb{E}_{T}>150 \mathrm{GeV}, M_{T}>150 \mathrm{GeV}, M_{T 2}^{W}>173 \mathrm{GeV}$.

where the basic cuts about $p_{T}$ and $\eta$ on leptons and jets are from the ATLAS report[2]. In order to improve the signal sensitivity, we increase the values of ATLAS cut $\mathbb{E}_{T}$ from 100 $\mathrm{GeV}$ to $150 \mathrm{GeV}$ to further suppress the semi-leptonic $t \bar{t}$ background and use the new cut $M_{T 2}^{W}>173 \mathrm{GeV}$ to reduce the di-leptonic $t \bar{t}$ background in our calculations.
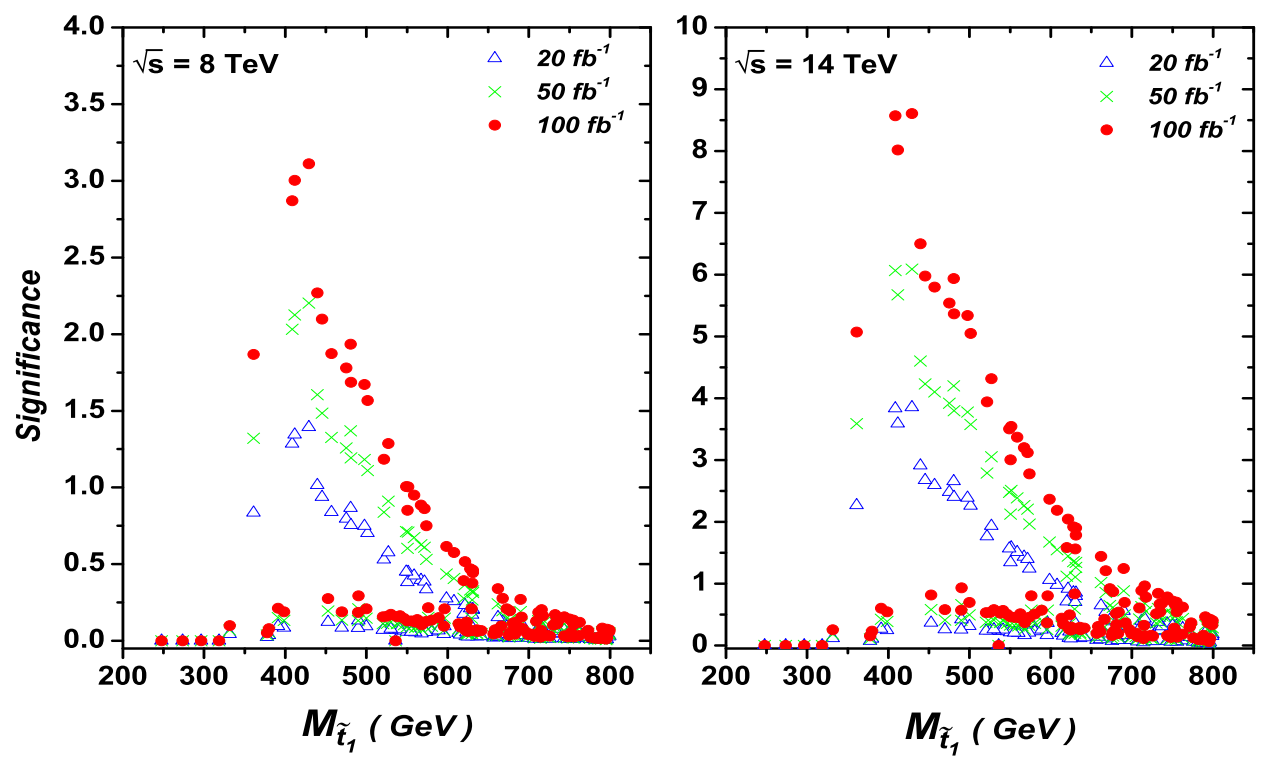

FIG. 6: The significance of stop pair production $p p \rightarrow \tilde{t}_{1} \tilde{t}_{1}^{*} \rightarrow t \tilde{\chi}_{1}^{0} \bar{t}_{1}^{0}$ for the surviving samples in the natural MSSM.

In Fig. 6 we show the significance of the surviving samples with $m_{\tilde{t}_{1}}<800 \mathrm{GeV}$. This figure indicates that the largest significance can be reached at $m_{\tilde{t}_{1}} \simeq 430 \mathrm{GeV}$ where the 

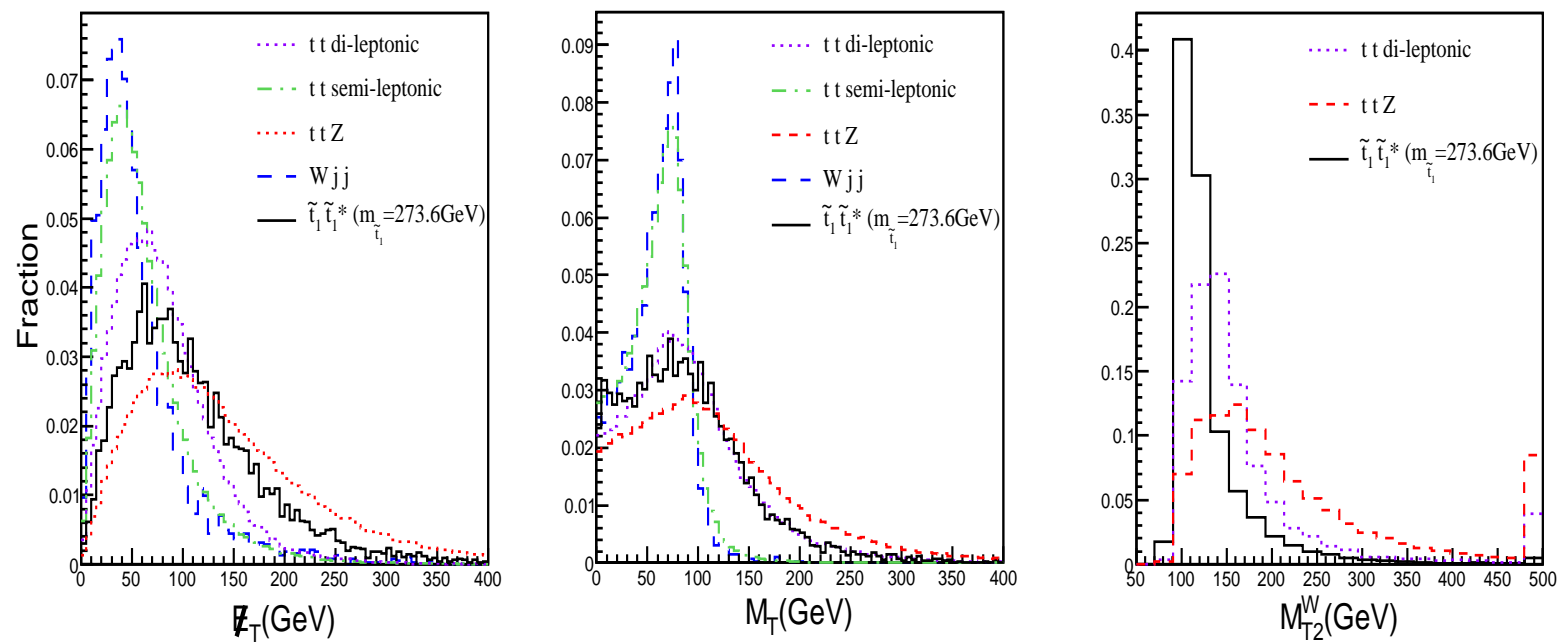

FIG. 7: Same as Fig.5, except that in plotting $M_{T 2}^{W}$ distribution we impose the cuts $\mathbb{E}_{T}>100$ $\mathrm{GeV}$ and $M_{T}>100 \mathrm{GeV}$ on the events.

significance is about 1.5 for $\sqrt{s}=8 \mathrm{TeV}$ with $20 \mathrm{fb}^{-1}$ luminosity and 8.5 for $\sqrt{s}=14 \mathrm{TeV}$ with $100 \mathrm{fb}^{-1}$ luminosity, and with the increase of the stop mass, the significance drop by one half for $m_{\tilde{t}_{1}} \simeq 500 \mathrm{GeV}$ mainly due to the reduction of the production rate. Our results are not as optimistic as those in [20, 21, 24] because we have taken into account the branch ratio of $\tilde{t}_{1} \rightarrow t \tilde{\chi}_{1}^{0}$. Fig, 6 also indicates that there are two branches for the significance in the mass region $320 \mathrm{GeV}<m_{\tilde{t}_{1}}<600 \mathrm{GeV}$. We checked that the upper branch corresponds to high branching ratio of $\tilde{t}_{1} \rightarrow t \tilde{\chi}_{1}^{0}$, which varies from $42.1 \%$ to $57.7 \%$ and results in a large signal rate, while the lower branch corresponds to a small ratio due to the competition of the decay mode $\tilde{t}_{1} \rightarrow t \tilde{\chi}_{1}^{0}$ with $\tilde{t}_{1} \rightarrow b \tilde{\chi}_{1}^{+}$and $\tilde{t}_{1} \rightarrow t \tilde{\chi}_{2}^{0}$.

Above analysis implies that, in order to fully explore the parameter space of the natural MSSM in stop detection, the decay mode $\tilde{t}_{1} \rightarrow b \tilde{\chi}_{1}^{+}$should also be considered. So we next consider the process

$$
p p \rightarrow \tilde{t}_{1} \tilde{t}_{1}^{*} \rightarrow\left(b \tilde{\chi}_{1}^{+}\right)\left(\bar{b} \tilde{\chi}_{1}^{-}\right) \rightarrow\left(b \ell^{+} \nu \tilde{\chi}_{1}^{0}\right)\left(\bar{b} j j \tilde{\chi}_{1}^{0}\right) \text { or }\left(b j j \tilde{\chi}_{1}^{0}\right)\left(\bar{b} \ell^{-} \bar{\nu} \tilde{\chi}_{1}^{0}\right)
$$

Same as in Fig [5, we show the distributions of the three variables in Fig 7 for the benchmark point $m_{\tilde{t}_{1}}=273.6 \mathrm{GeV}, m_{\tilde{\chi}_{1}^{+}}=163.5 \mathrm{GeV}$ and $m_{\tilde{\chi}_{1}^{0}}=156.3 \mathrm{GeV}$. Compared with the distribution in Fig, 5 , one can see that more signal events have lower values of $\mathbb{E}_{T}$ and low $M_{T}$, due to the relatively light $\tilde{t}_{1}$. Fig:7 also indicates the $M_{T 2}^{W}$ variable is helpless in suppressing the di-leptonic $t \bar{t}$ events and any cut on $M_{T 2}^{W}$ may hurt the signal greatly.

In Fig, we show the significance of the surviving samples for the process in Eq.(6). In 
order to keep more signal events, here we relax the cuts of $\mathbb{E}_{T}$ and $M_{T}$ used for the process in Eq.(5) as follows:

$$
\mathbb{E}_{T}>100 \mathrm{GeV}, \quad \mathrm{M}_{\mathrm{T}}>100 \mathrm{GeV}
$$

From this figure, one can learn that, due to the large stop pair production rate, the significance for $m_{\tilde{t}_{1}}=250 \mathrm{GeV}$ may reach 7 for $\sqrt{s}=8 \mathrm{TeV}$ with $20 \mathrm{fb}^{-1}$ luminosity and 64 for $\sqrt{s}=14 \mathrm{TeV}$ with $100 \mathrm{fb}^{-1}$ luminosity, but for $m_{\tilde{t}_{1}}=400 \mathrm{GeV}$, the maximum value drops to 1.5 and 10 respectively.
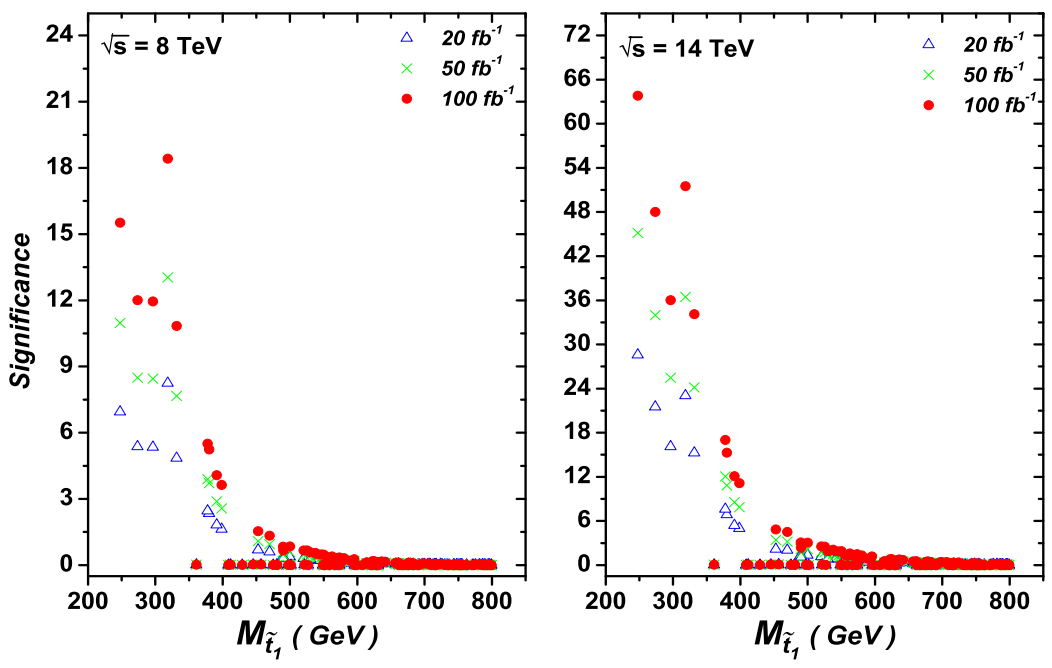

FIG. 8: The significance of stop pair production $p p \rightarrow \tilde{t}_{1} \tilde{t}_{1}^{*} \rightarrow\left(b \tilde{\chi}_{1}^{+}\right)\left(\bar{b} \tilde{\chi}_{1}^{-}\right)$for the surviving samples in the natural MSSM.

Finally, we summary the significance of the direct stop pair production with the above two decay modes of $\tilde{t}_{1}$ for $\sqrt{s}=14 \mathrm{GeV}$ and $100 \mathrm{fb}^{-1}$ luminosity. The results are displayed in Fig 9 where only the maximal significance under each cut is shown. This figure indicates that, for $m_{\tilde{t}_{1}}<400 \mathrm{GeV}$, detecting the stop pair production through the chargino decay is more effective, while for $400 \mathrm{GeV} \leq m_{\tilde{t}_{1}} \leq 450 \mathrm{GeV}$ the neutralino decay is more effective. This figure also indicates that the LHC can discover $\tilde{t}_{1}$ predicated in nature MSSM up to $450 \mathrm{GeV}$. If no excess events were observed at the LHC, the 95\% C.L. exclusion limits of the stop masses can go up to around $537 \mathrm{GeV}$ no matter what decay modes of the stop in the natural MSSM. 


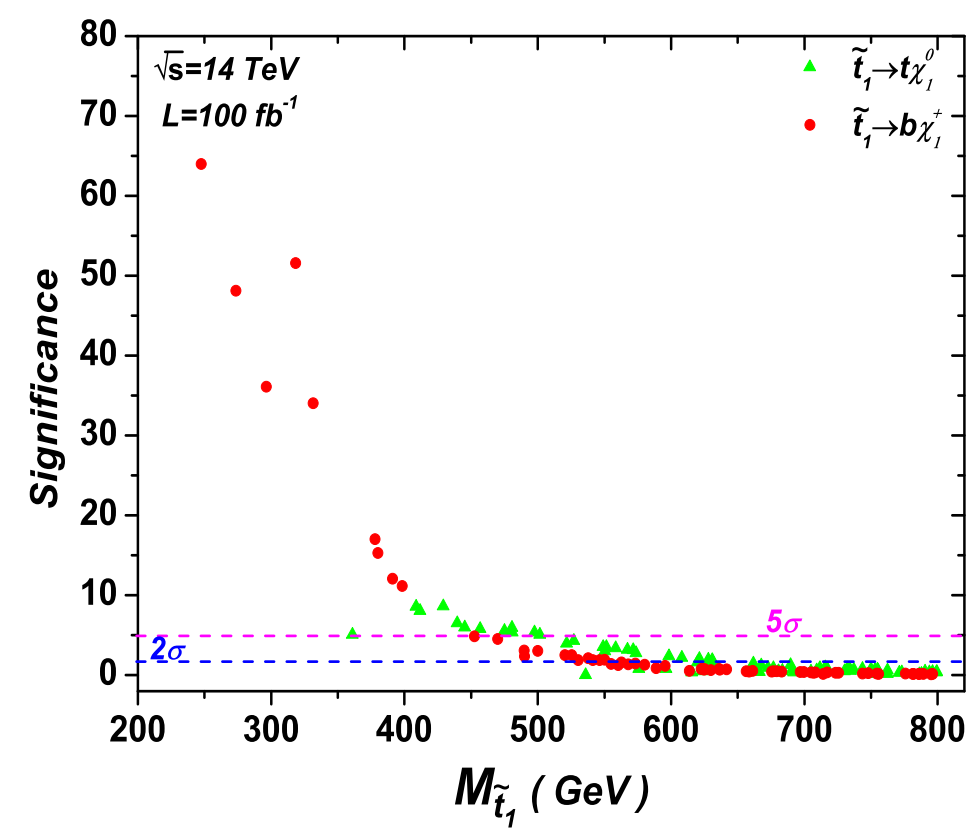

FIG. 9: The maximal significance in Eq.(51) or Eq.(6) as a function of the stop mass. The bullets (red) are obtained in Eq.(66) while the triangles (green) are obtained in Eq.(5).

\section{CONCLUSION}

In this work we studied the direct stop pair production at the LHC in the natural MSSM. We scanned over the corresponding parameter space by considering various experimental constraints and then in the allowed parameter space we examined the observability of the direct stop pair production at the LHC through the semi-leptonic analysis. We focused on the following two channels

$$
\begin{aligned}
& p p \rightarrow \tilde{t}_{1} \tilde{t}_{1}^{*} \rightarrow\left(t \tilde{\chi}_{1}^{0}\right)\left(\bar{t} \tilde{\chi}_{1}^{0}\right) \rightarrow\left(b \ell^{+} \nu \tilde{\chi}_{1}^{0}\right)\left(\bar{b} j j \tilde{\chi}_{1}^{0}\right) \text { or }\left(b j j \tilde{\chi}_{1}^{0}\right)\left(\bar{b} \ell^{-} \bar{\nu} \tilde{\chi}_{1}^{0}\right), \\
& p p \rightarrow \tilde{t}_{1} \tilde{t}_{1}^{*} \rightarrow\left(b \tilde{\chi}_{1}^{+}\right)\left(\bar{b} \tilde{\chi}_{1}^{-}\right) \rightarrow\left(b \ell^{+} \nu \tilde{\chi}_{1}^{0}\right)\left(\bar{b} j j \tilde{\chi}_{1}^{0}\right) \text { or }\left(b j j \tilde{\chi}_{1}^{0}\right)\left(\bar{b} \ell^{-} \bar{\nu} \tilde{\chi}_{1}^{0}\right),
\end{aligned}
$$

and performed detailed Monte Carlo simulations about the signals and backgrounds. We found that for $m_{\tilde{t}_{1}}<400 \mathrm{GeV}$ the second channel is better while for $400 \mathrm{GeV} \leq m_{\tilde{t}_{1}} \leq$ $450 \mathrm{GeV}$ the first channel is better. We also found that the LHC with $\sqrt{s}=14 \mathrm{TeV}$ and $100 \mathrm{fb}^{-1}$ luminosity is capable of discovering $\tilde{t}_{1}$ predicated in nature MSSM up to $450 \mathrm{GeV}$. If no excess events were observed at the LHC, the $95 \%$ C.L. exclusion limits of the stop masses can reach around $537 \mathrm{GeV}$ in the natural MSSM. 


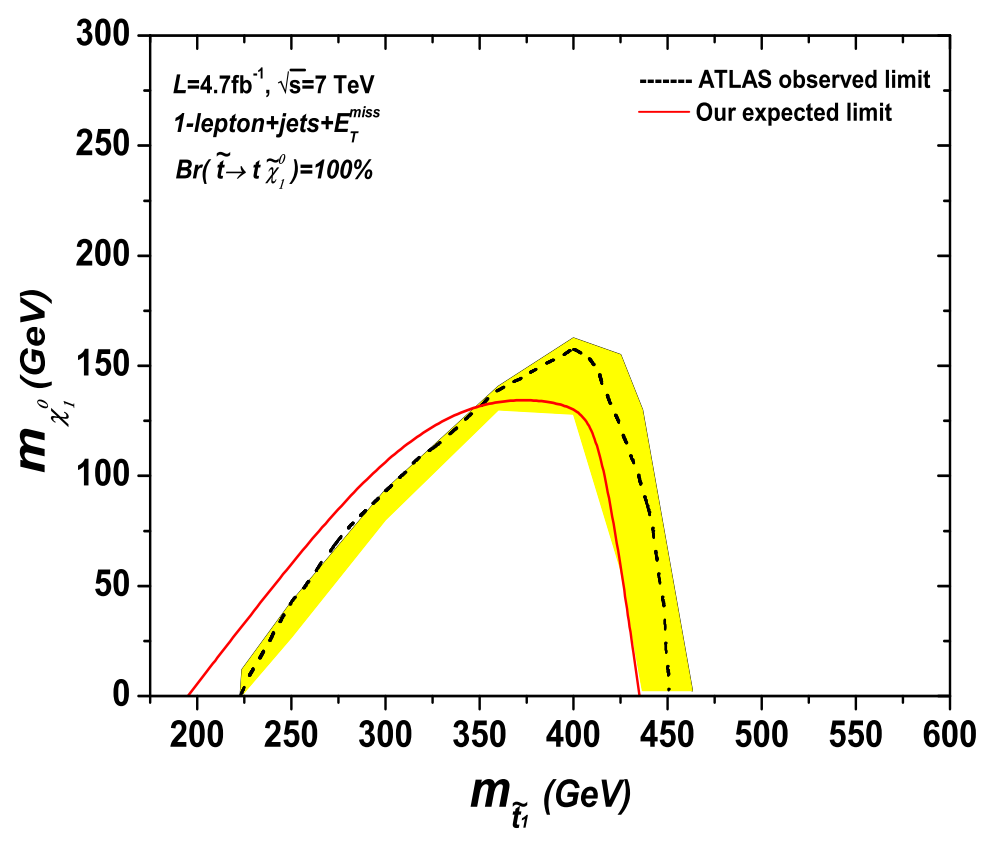

FIG. 10: The ATLAS observed and our expected exclusion limit in the plane of $m_{\tilde{\chi}_{1}^{0}}$ versus $m_{\tilde{t}_{1}}$, assuming $B R\left(\tilde{t}_{1} \rightarrow t \tilde{\chi}_{1}^{0}\right)=100 \%$.

\section{Note Added}

Very recently, the ATLAS collaboration reported the result of the direct searching for the stop pair production base on $4.7 \mathrm{fb}^{-1}$ of data[51]. We validated our simulation by reproducing the ATLAS exclusion limit according to the assumptions and cuts in the report as follows:

- One isolated electron or muon passing 'tight' selection criteria;

- Electrons $E_{T}>25 \mathrm{GeV}$ and $|\eta|<2.47$;

- Muon: $E_{T}>20 \mathrm{GeV}$ and $|\eta|<2.4$.

- Four or more jets with $|\eta|<2.5$ and $P_{T}>80,60,40$ and $25 \mathrm{GeV}$, and at least one jet to be identified as a b-jet;

- $\Delta \phi_{\min }>0.8$,where $\Delta \phi_{\min }$ is the minimum azimuthal separation between the two highest $P_{T}$ jets and the missing transverse momentum direction; 
- The jet-jet pair having invariant mass $>60 \mathrm{GeV}$ and the smallest $\Delta R$ is selected to form the hadronically decaying $W$ boson. The mass $m_{j j j}$ is reconstructed including a third jet closest in $\Delta R$ to the hadronic $W$ boson momentum vector and $130 \mathrm{GeV}$ $<m_{j j j}<205 \mathrm{GeV}$ is required;

- $E_{T}^{\text {miss }}>150 \mathrm{GeV}, E_{T}^{\text {miss }} / \sqrt{H_{T}}>7 \mathrm{GeV}^{1 / 2}$ and $m_{T}>120 \mathrm{GeV}$;

- Events are rejected if they contain additional leptons passing looser selection criteria. Here we treat the looser selection criteria as $P_{T}>15 \mathrm{GeV}$.

- The branch ratio of $\tilde{t}_{1} \rightarrow t \tilde{\chi}_{1}^{0}$ is assumed to be $100 \%$.

In Fig.10, we display the expected exclusion limit from our simulation. Considering the differences between the fast simulation and full detector simulation, we can see that our result is consistent with the ATLAS exclusion limit within the reasonable error range. We also expect our result can be improved by the simultaneous fits method used by ATLAS for five signal regions and three control regions, however, which is beyond the scope of our simulation. It should be noted that the above stop masses limits can be avoided in our study, since the stop decays with a mixture of the branching ratios.

\section{Acknowledgement}

Lei Wu thanks Xerxes Tata, Zijun Xu and Qiang Li for helpful discussion about the natural SUSY and MG/ME, and appreciates the organizers and lecturers at the KIAS school on MadGraph for LHC physics simulation (Oct. 24-29, 2011, KIAS, Soul). This work was supported in part by the National Natural Science Foundation of China (NNSFC) under grant Nos. 10821504, 11135003, 10775039, 11075045, by Specialized Research Fund for the

Doctoral Program of Higher Education with grant No. 20104104110001, and by the Project of Knowledge Innovation Program (PKIP) of Chinese Academy of Sciences under grant No. KJCX2.YW.W10.

[1] ATLAS Collaboration, ATLAS-CONF-2012-093; CMS Collaboration,CMS-PAS-HIG-12-020; G. Aad et al. [ATLAS Collaboration], Phys. Lett. B 710, 49 (2012); S. Chatrchyan et al. 
[CMS Collaboration], Phys. Lett. B 710, 26 (2012).

[2] G. Aad et al. [ATLAS Collaboration], Phys. Rev. Lett. 108, 041805 (2012).

[3] G. Aad et al. [ATLAS Collaboration], Phys. Rev. D 85, 112006 (2012); Phys. Rev. Lett. 108, 241802 (2012); Phys. Rev. Lett. 108, 181802 (2012).

[4] V. M. Abazov et al. [D0 Collaboration], Phys. Lett. B 710, 578 (2012); Phys. Lett. B 696, 321 (2011); T. Aaltonen et al. [CDF Collaboration], Phys. Rev. Lett. 106, 191801 (2011); Phys. Rev. Lett. 107, 191803 (2011).

[5] S. Chatrchyan et al. [CMS collaboration], Phys. Rev. Lett. 107, 221804 (2011); G. Aad et al. [ATLAS collaboration], arXiv:1109.6572 [hep-exp].

[6] M. Carena, S. Gori, N. R. Shah and C. E. M. Wagner, JHEP 1203, 014 (2012).

[7] see, e.g., J. Cao et al. Phys. Lett. B 710, 665 (2012); H. Baer, V. Barger and A. Mustafayev, JHEP 1205, 091 (2012); L. Aparicio, D. G. Cerdeno and L. E. Ibanez, JHEP 1204, 126 (2012); J. Ellis, K. A. Olive and K. A. Olive, Eur. Phys. J. C 72, 2005 (2012); C. Balazs et al., arXiv:1205.1568. A. Fowlie et. al., arXiv:1206.0264;

[8] J. Cao, et al., JHEP 1203, 086 (2012).

[9] see, e.g., U. Ellwanger, JHEP 1203, 044 (2012); J. F. Gunion, Y. Jiang and S. Kraml, Phys. Lett. B 710, 454 (2012); U. Ellwanger and C. Hugonie, arXiv:1203.5048 [hep-ph]; D. A. Vasquez et. al., Phys. Rev. D 86, 035023 (2012).

[10] R. Arnowitt and P. Nath, Phys. Rev. D 46, 3981 (1992).

[11] S. F. King, M. Muhlleitner and R. Nevzorov, Nucl. Phys. B 860, 207 (2012).

[12] P. Huet and A. E. Nelson, Phys. Rev. D 53 (1996) 4578; M. Carena, G. Nardini, M. Quiros and C. E. M. Wagner, Nucl. Phys. B 812 (2009) 243; Y. Li, S. Profumo and M. Ramsey-Musolf, Phys. Lett. B 673 (2009) 95.

[13] K. Griest and D. Seckel, Phys. Rev. D 43 (1991) 3191; C. Boehm, A. Djouadi and M. Drees, Phys. Rev. D62 (2000) 035012.

[14] C. Brust, A. Katz, S. Lawrence and R. Sundrum, JHEP 1203, 103 (2012).

[15] D. Feldman, G. Kane, E. Kuflik and R. Lu, Phys. Lett. B 704, 56 (2011); H. Baer et al., JHEP 1010, 018 (2010); A. Cohen, D. B. Kaplan and A. Nelson, Phys. Lett. B 388, 588 (1996); M. Dine, A. Kagan and S. Samuel, Phys. Lett. B 243, 250 (1990).

[16] J. L. Feng and D. Sanford, arXiv:1205.2372 [hep-ph]; G. Bhattacharyya and T. S. Ray, JHEP 1205, 022 (2012); S. Krippendorf, H. P. Nilles, M. Ratz and M. W. Winkler, Phys. Lett. B 
712, 87 (2012); B. C. Allanach and B. Gripaios, JHEP 1205, 062 (2012); S. Akula, M. Liu, P. Nath and G. Peim, Phys. Lett. B 709, 192 (2012); L. J. Hall, D. Pinner and J. T. Ruderman, JHEP 1204, 131 (2012); M. Asano, H. D. Kim, R. Kitano and Y. Shimizu, JHEP 1012, 019 (2010); R. Kitano and Y. Nomura, Phys. Rev. D 73, 095004 (2006); J. Hisano, K. Kurosawa and Y. Nomura, Nucl. Phys. B 584, 3 (2000); J. L. Feng, K. T. Matchev and T. Moroi, Phys. Rev. D 61, 075005 (2000); K. L. Chan, U. Chattopadhyay and P. Nath, Phys. Rev. D 58, 096004 (1998); G. W. Anderson, D. J. Castano and A. Riotto, Phys. Rev. D 55, 2950 (1997).

[17] H. Baer, V. Barger, P. Huang and X. Tata, JHEP 1205, 109 (2012).

[18] M. Papucci, J. T. Ruderman and A. Weiler, arXiv:1110.6926 [hep-ph];

[19] see, e.g., A. Choudhury and A. Datta, JHEP 1206, 006 (2012)? K. Huitu, L. Leinonen and J. Laamanen, Phys. Rev. D 84, 075021 (2011); Y. Kats and D. Shih, JHEP 1108, 049 (2011); S. Bornhauser, M. Drees, S. Grab and J. S. Kim, Phys. Rev. D 83, 035008 (2011); N. Bhattacharyya, A. Choudhury and A. Datta, Phys. Rev. D 84, 095006 (2011); D. Casadei, R. Konoplich and R. Djilkibaev, Phys. Rev. D 82, 075011 (2010); K. Rolbiecki, J. Tattersall and G. Moortgat-Pick, Eur. Phys. J. C 71, 1517 (2011); M. Perelstein and A. Weiler, JHEP 0903, 141 (2009); T. Han, R. Mahbubani, D. G. E. Walker and L. -T. Wang, JHEP 0905, 117 (2009); M. Carena, A. Freitas and C. E. M. Wagner, JHEP 0810, 109 (2008); S. Kraml and A. R. Raklev, Phys. Rev. D 73, 075002 (2006); T. Han at al., Phys. Rev. D 70, 055001 (2004). A. Bartl et al., Phys. Lett. B 573, 153 (2003); J. Hisano, K. Kawagoe and M. M. Nojiri, Phys. Rev. D 68, 035007 (2003); J. Hisano, K. Kawagoe, R. Kitano and M. M. Nojiri, Phys. Rev. D 66, 115004 (2002); J. M. Yang and B. -L. Young, Phys. Rev. D 62, 115002 (2000);

[20] D. E. Kaplan, K. Rehermann and D. Stolarski, JHEP 1207, 119 (2012).

[21] T. Plehn, M. Spannowsky and M. Takeuchi, JHEP 1208, 091 (2012); Phys. Rev. D 85, 034029 (2012); JHEP 1105, 135 (2011); T. Plehn and M. Spannowsky, arXiv:1112.4441; T. Plehn et al., JHEP 1010, 078 (2010); T. Plehn, G. P. Salam and M. Spannowsky, Phys. Rev. Lett. 104, 111801 (2010).

[22] J. Thaler and K. Van Tilburg, JHEP 1202, 093 (2012); J. Thaler and K. Van Tilburg, JHEP 1103, 015 (2011); J. Thaler and L. -T. Wang, JHEP 0807, 092 (2008).

[23] K. Rehermann and B. Tweedie, JHEP 1103, 059 (2011); M. Jankowiak and A. J. Larkoski, JHEP 1106, 057 (2011); L. G. Almeida et al., Phys. Rev. D 82, 054034 (2010); Phys. Rev. D 79, 074012 (2009); D. E. Kaplan et al., Phys. Rev. Lett. 101, 142001 (2008). 
[24] Y. Bai, H. -C. Cheng, J. Gallicchio and J. Gu, arXiv:1203.4813 [hep-ph].

[25] Z. Han, A. Katz, D. Krohn and M. Reece, JHEP 1208, 083 (2012).

[26] M. Drees, M. Hanussek and J. S. Kim, arXiv:1201.5714 [hep-ph];

[27] D. S. M. Alves et al., arXiv:1205.5805 [hep-ph];

[28] C. Brust, A. Katz and R. Sundrum, JHEP 1208, 059 (2012); H. -T. Wei, et al., JHEP 1107, 003 (2011); N. Desai and B. Mukhopadhyaya, JHEP 1010, 060 (2010).

[29] X. -J. Bi, Q. -S. Yan and P. -F. Yin, Phys. Rev. D 85, 035005 (2012);

[30] M. Frank et al., JHEP 0702, 047 (2007); G. Degrassi et al., Eur. Phys. J. C 28, 133 (2003); S. Heinemeyer, W. Hollik and G. Weiglein, Comput. Phys. Commun. 124, 76 (2000); Eur. Phys. J. C 9, 343 (1999).

[31] P. Bechtle et al., Comput. Phys. Commun. 182, 2605 (2011); Comput. Phys. Commun. 181, 138 (2010).

[32] K. Ishiwata, N. Nagata and N. Yokozaki, Phys. Lett. B 710, 145 (2012).

[33] J. Rosiek et al., Comput. Phys. Commun. 181, 2180 (2010); A. Crivellin, L. Hofer and J. Rosiek, JHEP 1107, 017 (2011).

[34] J. Cao and J. M. Yang, JHEP 0812, 006 (2008).

[35] J. Dunkley et. al. [WMAP Collaboration], Astrophys. J. Suppl. 180, 306 (2009)

[36] G. Belanger et al., Comput. Phys. Commun. 182, 842 (2011).

[37] M. S. Carena et al., Eur. Phys. J. C 26, 601 (2003).

[38] J. Cao et al., Phys. Lett. B 703, 462 (2011);

[39] M. Carena et al., arXiv:1205.5842 [hep-ph].

[40] M. R. Buckley and D. Hooper, arXiv:1207.1445 [hep-ph].

[41] A. Djouadi, Phys. Lett. B 435, 101 (1998); hep-ph/9901237).

[42] W. Beenakker et al., Nucl. Phys. B 515, 3 (1998);

[43] J. Pumplin et al., JHEP 0602, 032 (2006).

[44] M. Muhlleitner, A. Djouadi and Y. Mambrini, Comput. Phys. Commun. 168, 46 (2005).

[45] N. Kidonakis, Phys. Rev. D 82, 114030 (2010); V. Ahrens et al., Phys. Lett. B 703,135 (2011); M. Cacciari et al., Phys. Lett. B 710, 612 (2012); S. Moch, P. Uwer and A. Vogt, arXiv:1203.6282,

[46] J. Alwall et al., JHEP 1106, 128 (2011).

[47] T. Sjostrand, S. Mrenna and P. Z. Skands, JHEP 0605, 026 (2006). 
[48] S. Ovyn, X. Rouby and V. Lemaitre, arXiv:0903.2225 [hep-ph].

[49] M. Cacciari, G. P. Salam and G. Soyez, JHEP 0804, 063 (2008).

[50] F. Caravaglios, M. L. Mangano, M. Moretti and R. Pittau, Nucl. Phys. B 539, 215 (1999).

[51] G. Aad et al. [ATLAS Collaboration], arXiv:1208.2590 [hep-ex]. 